\title{
Applications Research of Safety Accidents in Chemical Production Based on GIS Technology
}

\author{
Junyan Huang \\ Chongqing Vocational Institute of Safety \& Technology, Chongqing, 404020, China
}

Keywords: GIS technology, Safety accidents, Chemical production

\begin{abstract}
The prevention and control of accidents in chemical industry involves decision-making from all sides. The traditional decision-making method is based on personal knowledge, experience and judgment with great subjectivity. Therefore, it is particularly important to explore new method to deal with chemical safety accidents and improve the rationality of emergency evacuation decision-making. This paper explores the main functions of GIS technology in safety accidents management in chemical production and discusses the design principles, development environment and function realization of chemical safety production system based on GIS technology.
\end{abstract}

\section{Introduction}

Since the development and use of geographical information system (GIS) in Canada in the 1960s, the technology has been developing and its application has gradually expanded to all areas of social life [1]. Its development history is closely related to the development of computer technology and geographical science. With the rise of Web Service technology and its application in GIS, geographic information service is becoming more and more popular in various fields and industries. GIS is an information system based on geo-spatial database and using geo-model method to provide multi-spatial and dynamic geographic information timely for geographic research and geographic decision-making. It has been widely used in resource management, urban planning, emergency rescue and other fields. From the point of view of graphics, GI S is essentially a kind of digitized map or drawing which is convenient for computer processing and network transmission. It can input, edit, display, query, spatial analysis and output printing spatial data conveniently. It can also simulate various elements by using three-dimensional dynamic simulation technology to construct one. A realistic simulation space reveals the complex temporal relationships among various objects. GIS includes not only the spatial data of the distribution of various elements, but also the attribute data of these elements, such as pipe diameter, material, maintenance records, emergency plans and so on. Moreover, there is a close relationship between spatial data and attribute data. Spatial information and attribute information forms an organic whole. By retrieving spatial data, the corresponding attribute information can be queried, and by retrieving attribute data, the corresponding spatial information can be queried, which avoids the separation of graphics and corresponding attribute data, and brings great convenience to the overall management of enterprise resources. GIS system can make use of its spatial database and attribute database, combined with efficient algorithm for spatial analysis [2].

\section{Main Functions of GIS Technology in Safety Accidents Management in Chemical Production}

\subsection{Information Inquiry}

By making full use of the visualization function of GIS technology, the geographic location of the enterprises in the chemical industrial park can be marked on the map of GIS, and the geographic distribution of the enterprises can be grasped as a whole. When in an emergency state, the 
medium-sized incident enterprises can be selected to highlight the important safety information such as the general layout map, the surrounding environment diagram, the safety management team, the rescue force, the risk level, the major hazard sources and so on. The time consumed by searching for information in a rush mode. Combined with the emergency disposal process, the emergency resources of the emergency module are constructed as follows. After receiving the report of the enterprise's emergency, the District Command team must first report the information to the relevant departments according to the requirements, so the management information is essential in the emergency resources; then, when receiving the enterprise's emergency response and expanding the appeal, the District Command team needs to coordinate the public security firefighting, medical resources, emergency materials loading and unloading. For this reason, the emergency module should also associate medical resources, public security teams and emergency materials with geographic information. Finally, when the District Command team allocates emergency resources, it must be effectively guaranteed if it needs unusual social resources such as special equipment transportation, so is the transportation guarantee. The indispensable emergency resources in the emergency module. At the same time, in order to avoid secondary accidents caused by hazards near the accident site, the function of hazard source inquiry is added. The construction principle of hazard information on map is basically consistent with enterprise information. To realize the function of querying and supervising enterprise information, it is necessary to correlate this data with the basic information of enterprise module and hazard source module [3].

\subsection{Rescue Analysis}

In the process of emergency response, how to allocate the resources to the scene in an effective time after the command team has mastered the available emergency resources directly determines the benefits of emergency response. Through the previous research, the choice of rescue path determines the timeliness of resource allocation. For this reason, the module's rescue analysis focuses on path selection. The idea of path selection: firstly, the resources that need to be invoked are selected through the geographic information layer. The system will identify the geographical location of each resource as the starting point of the path, and then select the incident location on the map, that is, the default path end point of the system. Finally, the system will mark the path from each starting point to the end point through the colored line segment. Path and show the distance of each path in the menu window. The path calculation combines Dijkstra algorithm with the map of the traffic line to optimize the algorithm, to ensure that the path will not cross the building and other calculation errors. The combination of GIS software and application model can expand the function of GIS. By inputting relevant information, we can quickly process the data we need, and display it visually in the form of graphics and graphics visualization, which can provide objective and reasonable decision support for various problems in reality. This is assistant decision-making. Support system. Using this system, the key and useful information can be extracted from the massive emergency evacuation information of the park, and the data can be processed to assist the park managers in decision-making. The assistant decision-making system based on GIS can provide an assistant decision-making scheme by human-computer interaction through safety production data, accident model, knowledge base and field monitoring feedback information. It provides an environment for park emergency managers to analyze problems, simulate evacuation decision-making process and provide assistant decision-making scheme. By using relevant information resources and analysis tools, we can improve the decision-making level and quality of Park emergency managers [4].

\subsection{Accident Simulation}

Emergency module of the accident simulation, on the one hand, can simulate the situation of the accident development in the emergency state, help the District Command team to predict the change process of the impact of the accident; on the other hand, can clear the emergency evacuation route in the emergency drill forbidden zone, summarize the evacuation experience. At present, the application of accident simulation in GIS is mainly two-dimensional simulation, the main reason is that the numerical simulation itself needs a strong computing memory space, and requires a high degree of 
computing hardware; with the increase of dimensions, if the hardware conditions cannot meet the requirements, it is easy to cause system jam or even interruption because of the operation. For this reason, this paper also uses two-dimensional simulation to construct the accident simulation, in order to ensure the effect of accident simulation, while maintaining the stable operation of the geographical information system. Influenced by the accident, it may lead to ground communication interruption and traffic arteries being destroyed, which will affect the release, transmission and sharing of accident information. GIS has powerful spatial data processing, display and graphics output functions, combined with the corresponding Internet of Things technology, can effectively solve this problem. Computer simulation is to simulate the flow of people or traffic conditions in the evacuation process from the micro or macro level. It can be used to predict the evacuation time and evaluate the evacuation plan, but it does not consider the impact of accident consequences, resulting in the simulation results are still a certain gap with the actual situation, but also not suitable for evacuation planning. And overall optimization of transport strategy.

\section{System Development of Chemical Safety Production Based on GIS Technology}

\subsection{System Design Principles}

After fully investigating the system requirements and combining with the design principles, the component GIS technology is selected in the design of the open area hazard source management and emergency system. It can uniquely encode the entity and establish 1:N relationship; query association function based on SQL Server, Association attribute data and spatial data. As the representative of mature GIS technology, component GIS technology has rich practical results, which can lay a solid foundation for the safety and stability of the open area hazard management and emergency system design. In designing the management and emergency system of dangerous source through open area, the following points should be considered in combination with the requirements of users under the condition of adhering to the basic principles of software design. Technical route requirements: the platform of software must be based on Windows platform, and it has extensibility. The system network is built and accessed based on the local area network of the open area. Security and reliability: Require the system in the overall design, platform selection and application development to ensure that the system is safe, stable and reliable operation. Scalability: In the process of system design, we should adopt the idea of "object-oriented" design and the method of software engineering. At the same time, we should adopt the method of multi-tier architecture and platform to ensure that the system has perfect system management and good scalability, and can provide support for future application expansion. According to business needs, the corresponding expansion development space is reserved. Easy to maintain: Require the system structure design of service products to follow the development principles of software engineering, clear hierarchy, easy to understand. We should have perfect development technical documents and user documents to make the system easy to maintain, interface standardization and software design standardization.

\subsection{System Development Environment}

The system is developed by Arc GIS Engine component. Arc GIS Engine has the basic services of GIS. It can access many geographic data. The symbolic library provided by Arc GIS Engine can express thematic maps. It has the control of beautifying program interface. It can also use standard function and advanced function together. The system development environment is deployed by installing Arc GIS Engine development kit, Arc GIS Server 9.2 and SQL Server 2008. The system chooses $C_{-} \#$ as the development language, on the one hand, because many professional software related to GIS system is developed by C_\#, which reflects that C_\# is already a very mature development language in the field of GIS; on the other hand, C_\#, as an object-oriented development language, inherits the same powerful functions of $\mathrm{C}$ and $\mathrm{C}++$. When we use $\mathrm{C \#}$ to develop GIS system, we can gradually improve the development method and add various requirements, which greatly enhance the expansibility of the system. The actual distribution of important chemical regions 
and planning information for the next few years will be displayed intuitively to provide graphical information for the next planning. At the same time, statistics of business development information, analysis of the development of enterprises, the corresponding statistical chart. The actual output of major projects and the forecast before investment are analyzed and evaluated to provide decision-making basis for the formulation of development planning. Aiming at the characteristics of easily producing inflammable and explosive substances and high safety requirements in chemical production, emergency mechanism is established for key areas and key installations in chemical production, and corresponding plans are set up. The contingency plan subsystem records these plans into the system, which is convenient for users to inquire and learn.

\subsection{System Function Realization}

Accident Emergency Assistant Decision-making Module calls accident trend monitoring sub-module to simulate and monitor accident development trend. The concrete process is that the system automatically calls accident simulation database, dangerous source or dangerous process information according to real-time monitoring data, and carries out accident simulation and monitoring accident development trend in the background. The monitoring results are illustrated and written. Form display in the system interface: according to the serious situation and development trend of the accident, call emergency resource information database, GIS information database, the best rescue path calculation module to generate emergency rescue plan, the best rescue path, the best evacuation path: emergency rescue plan, the best rescue path, the best evacuation path The information such as scatter path is displayed on the scene of emergency command center platform and mobile GIS platform, and the relevant institutions and personnel are notified by short message. The auxiliary decision-making module of accident emergency mainly relies on GIS sub-module and assistant decision-making sub-module, and combines emergency plan database, emergency disposal knowledge database, emergency resource database, dangerous chemicals database and rescue laws and regulations to generate emergency assistant decision-making scheme, and through the real-time information feedback of accident scene to carry on the correct rescue scheme. When adjusting, a highly efficient and scientific emergency rescue work program is created, and the effectiveness and scientific of the plan is verified by accident consequence prediction and simulation, so as to ensure timely rescue and evacuation of the accident scene, reduce casualties and provide support for the improvement of the plan. When the park completes the enterprise information collection and is perfected into the information system, it can be linked to the emergency module through the enterprise coordinate position marked in the data collection. If you need to find the geographical location and detailed information of the enterprise on the thematic map, you can search the data by supervising the enterprise, and click on the target enterprise to highlight it on the map.

\section{Conclusion}

In this paper, the application of chemical safety accidents based on GIS is the main topic. A method of assistant decision-making for chemical park emergency evacuation based on GIS is proposed. Chemical industrial park emergency evacuation is a complex and ambitious project involving many aspects of decision-making. In this paper, only the scheme of assistant decision-making for the relatively important and urgent key decision-making content faced in the accident is given. If considering more comprehensively, we can further reduce the decision-making costs of emergency managers and improve the efficiency of decision-making.

\section{References}

[1] Li Jun, Li Qingqi, He Chengqiang, et al. Study on emergency response for leakage accident of dangerous chemical gas based on GIS [J]. Journal of Safety Science and Technology, 2017, 13(11): 66-72.

[2] Miu Pengfei, Liu Daoming, Tian Xin. Simulation analysis of LPG tank explosion based on GIS 
and its application in emergency disposal [J]. Journal of Liaoning Technical University (Natural Science), 2016, 35(9): 926-930.

[3] Chen Xuan, Chen Liangchao, Yang Jianfeng, et al. Refinery Equipment Corrosion and Protection Management System [J]. Pressure Vessel Technology, 2016, 33(4): 70-75.

[4] Yan Fengying, He Zenan, Zhang Qi. Study on Fire Risk Assessment for Chemical Industry Park Based on GIS [J]. Journal of Tianjin University (Social Sciences), 2016, 18(5): 418-423. 